Original Research

\title{
Assessment of Stability-Based Characterizations of North Carolina's Offshore Wind Resource Using a Nested Boundary Layer Method
}

\author{
Megan Schutt ${ }^{\dagger}, \ldots$, Harvey Seim ${ }^{\dagger, *}$
}

Marine Sciences, University of North Carolina at Chapel Hill, 3202 Venable Hall, Chapel Hill, NC, USA; E-Mails: meganschutt2643@gmail.com; hseim@email.unc.edu

¥ Current Affiliation: GE Renewables, Greenville SC, USA

$\dagger$ These authors contributed equally to this work.

* Correspondence: Harvey Seim; E-Mail: hseim@email.unc.edu

Academic Editor: Andrés Elías Feijóo Lorenzo

Special Issue: Offshore Wind Farms

Journal of Energy and Power Technology

2020, volume 2 , issue 2

doi:10.21926/jept.2002006
Received: April 10, 2020

Accepted: April 27, 2020

Published: April 30, 2020

\begin{abstract}
SODAR wind profiler measurements from a land-based site within $1000 \mathrm{~m}$ of the coastal ocean are compared with predicted wind profiles based on upwind conditions and a stability-based model. The thermally dynamic influence of the Gulf Stream near the site of measurement, Cape Hatteras, has been found to complicate wind speed estimation at height through its impacts on atmospheric stability and the resulting changes to wind shear. The COARE 3.0 algorithm was used to calculate a Monin-Obukhov Similarity Theory (MOST) scaling parameter, quantifying the degree of stability of the marine environment as well as the friction velocity and roughness length. Knowledge of upwind conditions aided in estimating the height of nested roughness and thermal internal boundary layers (IBLS) to identify a range of the measured wind profile corresponding to winds over the water. This work suggests that MOST through COARE 3.0 provides a good estimate of wind-at-height behaviour, with some exceptions. The layers identified in the IBL approximations indicate
\end{abstract}

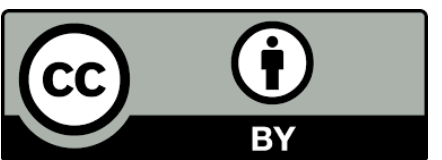

(C) 2020 by the author. This is an open access article distributed under the conditions of the Creative Commons by Attribution License, which permits unrestricted use, distribution, and reproduction in any medium or format, provided the original work is correctly cited. 
that (1) effects of stability can improve wind speed estimation when upwind conditions are well defined, but (2) unexpectedly high levels of wind shear make this method unreliable within unstable layers above stable layers. These results impact the estimate of the available wind resource, as well as other properties applicable to wind energy engineering, including shear and turbulence characteristics.

\section{Keywords}

Marine meteorology; offshore wind energy; wind profiles

\section{Introduction}

One way for renewable energy to become cost competitive with current fossil fuel sources is continued upscaling of wind turbine size [1]. Advancements of this nature, common to deployments offshore, require increased knowledge of wind speed profiles, especially over the range of height across the swept area of wind turbine blades. Marine wind systems have been shown to differ from land-based systems, especially regarding the influence of surface heating and lower atmospheric stability [2]. Monin-Obukhov Similarity Theory (MOST) has been used extensively to examine stability in the lower atmospheric boundary layer, but the historical focus on land-based systems lends a need for validation or refinement of empirically derived constants and functional forms for marine regions. The wind climatologies of coastal and offshore areas often lack the observations necessary to validate surface layer theory expectations. Past studies using a variety of wind-at-height estimation methodologies [3] have characterized North Carolina's offshore wind resource spatially and temporally, but none were able to employ wind profile measurements offshore for validation of these methods.

With wind energy area (WEA) lease block sales moving forward recently off several states in the US [4], the importance of adequately characterizing the available resource in the blocks is a necessary concern. In coastal North Carolina, the spatial thermal gradients at the sea surface created by the conflux of the Gulf Stream (GS), Mid-Atlantic Bight (MAB) waters, and SouthAtlantic Bight $(S A B)$ coastal waters can be large $\left(>15^{\circ} \mathrm{C}\right)$, as seen in Figure 1 . This spatial heterogeneity in sea surface temperature (SST) has been shown to induce both spatially and temporally varying changes in lower atmospheric stability and turbulence characteristics [5]. This geographic area is therefore an interesting, yet complicated, region to examine the impact of these spatial gradients on the lower atmospheric boundary layer $(A B L)$.

Presented in this study is an analysis of SODAR wind profiles measured in coastal North Carolina, addressing the vertical structure and predictability of wind profiles in the presence of large SST gradients. It remains difficult to collect long-term and highly resolved wind profiles over the water; as such, in this work, the site of measurement is a narrow strip of land between a large, shallow sound and the open ocean. While spatial heterogeneities and distance from the BOEMidentified WEAs make analysis at this location non-ideal, these are at present among the best observations available to assess marine wind profiles in the region. We estimate the height of internal boundary layers (IBLS) developing as a consequence of land-based roughness step changes and sea surface thermal gradients. Using this height estimate, a vertical range of the wind 
profile was identified that should retain characteristics of the upwind marine environment. These measured wind profiles are used in conjunction with near surface conditions in the open ocean as measured by buoys assumed to represent the upwind environment. A surface-based flux algorithm, COARE 3.0 [6], was used to quantify stability with these environmental measurements using MOST, and model the resulting wind profiles expected due to the stability regime. We then examine the skill of the model in depicting variations in wind speed, wind shear, and turbulence. This method, assessing the degree to which a surface layer model incorporating MOST can accurately represent the coastal marine boundary layer, had moderate skill in predicting wind speeds and did not explain the large and variable observed shear within the oceanic wind profiles when unstable conditions prevailed. Possible explanations for the inability of the model to explain the observations are discussed. It would appear that further study must be done with wind profile measurements over the ocean to both validate the levels of observed wind shear, as well as investigate the environmental conditions, e.g. air-sea heat exchange, mesoscale weather features, that lead to the observed conditions.

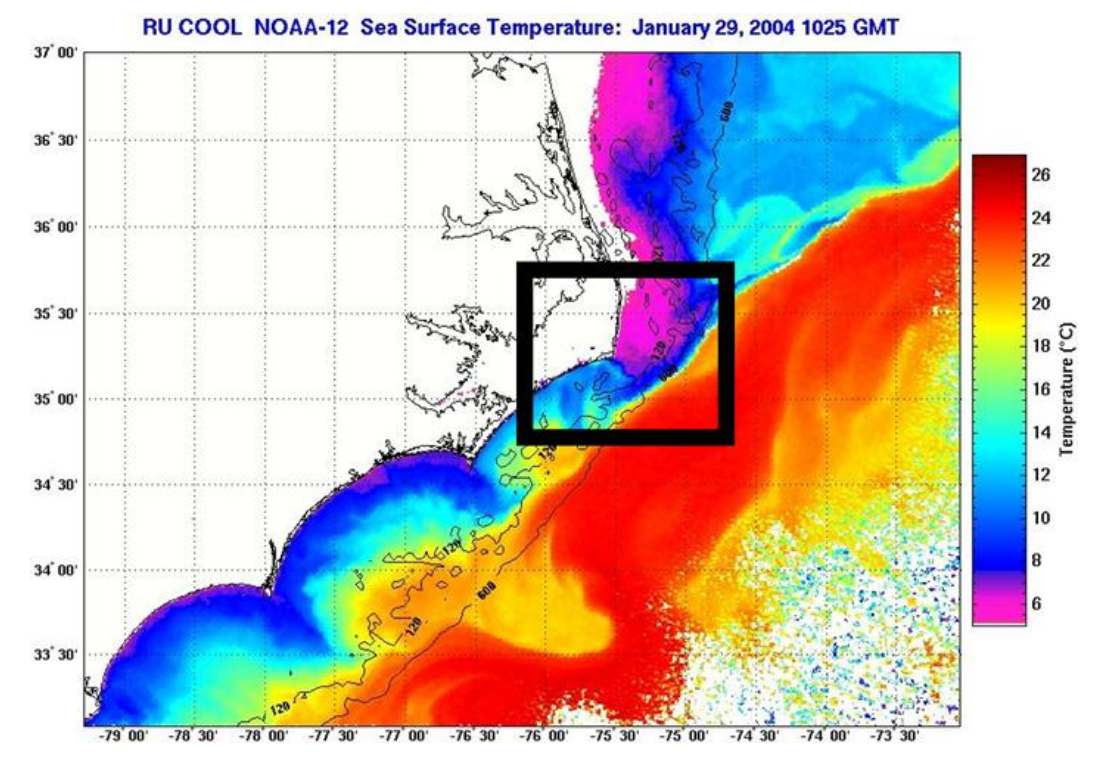

Figure 1 Satellite AVHRR SST image, showing strong thermal gradients between Gulf Stream and coastal waters (image c/o Rutgers COOL [7]). Black box outlines the study area and evidences the strong thermal surface gradients between GS, MAB and SAB waters.

\section{Materials and Methods}

A SODAR wind profiler (SCINTEC SFAS) stationed at Billy Mitchell airport, near Cape Hatteras, North Carolina was used to obtain hourly wind profiles. This site, a rural airport used by small aircraft, lies on a narrow island bounded to the south and east by the Atlantic Ocean and to the north by Pamlico Sound (Figure 2). The profiler is a compact phased array design and was configured to collect observations between $10 \mathrm{~m}$ and $200 \mathrm{~m}$ at $5 \mathrm{~m}$ increments every 30 minutes; uncertainties of individual horizontal velocity estimates are $<0.5 \mathrm{~m} / \mathrm{s}$. During the deployment from 2012-2014 there was varying temporal and spatial coverage due to instrument properties, environmental conditions and at times, instrument failures. The monthly mean wind profiles using only measurements where winds were coming from the ocean side of Hatteras Island exhibit a 
somewhat seasonal trend in profile shape and mean wind speed (Figure 3). However, the expected decrease in wind speed and wind shear in warmer months, due to more uniform SSTs, is not immediately evident with these measurements. Of particular interest is the persistent lowlevel maximum, present in nearly all months.

Two buoys located in Raleigh Bay (NDBC 41025 and 41063, Figure 2) provided wind speed and direction, surface air and water temperature, humidity and air pressure measurements during times when the SODAR was active. Surface wave measurements were also collected, as were current profiles at the 41063 buoy location.

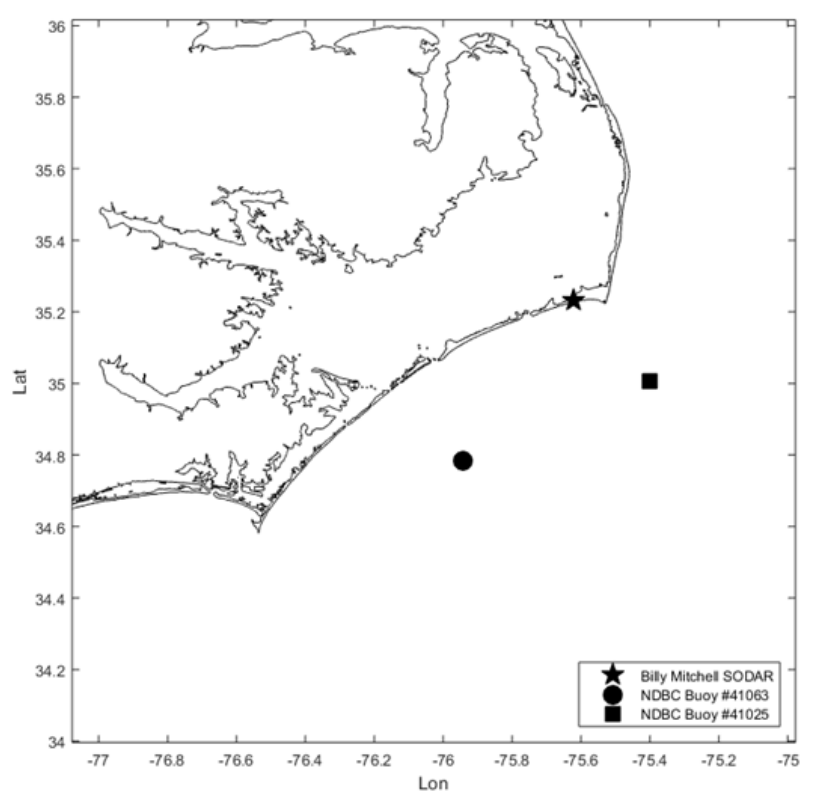

Figure 2 Locations of data sources for this analysis, relative to the NC coastline: Billy Mitchell SODAR on Hatteras Island, NDBC Buoy \#41063 in Raleigh Bay, and NDBC Buoy \#41025 on Diamond Shoals.
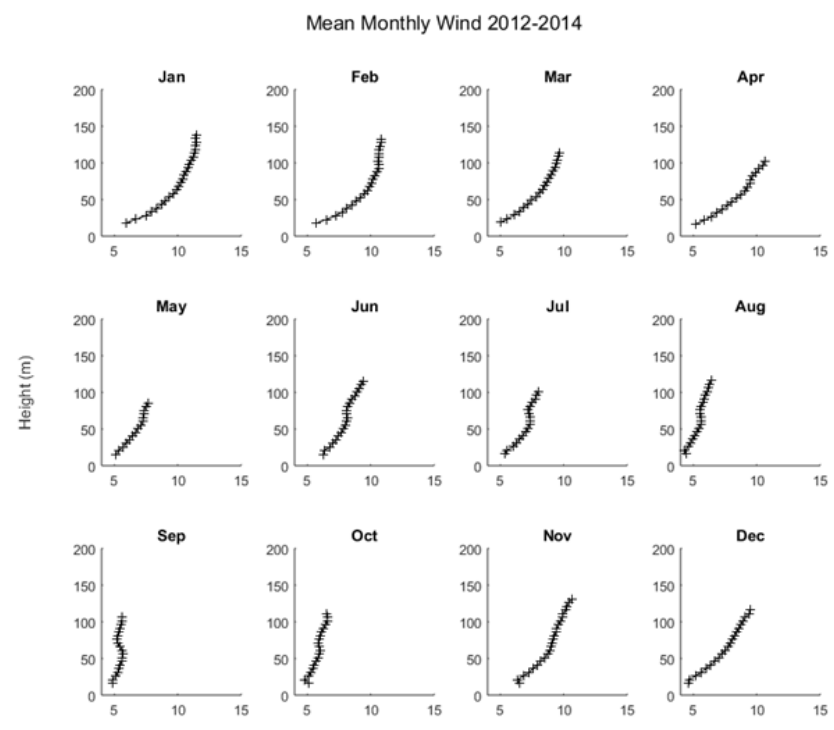

Wind Speed $(\mathrm{m} / \mathrm{s})$

Figure 3 Monthly mean wind speed profiles over three years. Time averages are formed for each vertical bin $(5 \mathrm{~m})$ where there is $80 \%$ coverage available. 


\subsection{IBL Algorithm}

The lower ranges of these wind profiles are influenced by land-based roughness elements. While higher regions of the profile may also be influenced indirectly by flow over land, they likely remain associated with regions of the ocean upwind of the SODAR. Observations from buoys in these regions can characterize the upwind environment, and be used to inform an analysis of the impact of stability on the range of the wind profile associated with that region. Identification of the IBLs developing as a response to the spatially and temporally heterogeneous surface characteristics (changes in roughness and thermal conditions) is an integral component to the analysis of the measured wind profiles, as only a particular vertical range may be indicative of the upwind region where the buoys are located and environmental conditions are measured. Heights of Roughness IBLS (RIBLS), $\delta_{R I B L}$, were defined using the formulation [8]

$$
\delta_{R I B L}=z_{01}\left[0.75+0.03 \ln \left(\frac{z_{02}}{z_{01}}\right)\right] *\left(\frac{x}{z_{01}}\right)^{0.8}
$$

where $z_{01}$ is the aerodynamic roughness length of the first surface, $z_{02}$ is the aerodynamic roughness length of the second surface, $x$ is the horizontal fetch over the second surface since the step change, and $z_{01}>z_{02}$, where aerodynamic roughness lengths are taken from a $250 \mathrm{~m}$ resolution AWS TrueWind product made for the state of North Carolina. Wind measurements within this RIBL will not be used in analysis of any of the wind profiles in this work, as they are associated with land conditions, as opposed to marine conditions. Heights of Thermal IBLS (TIBLS), $\delta_{T I B L}$, due to stark step changes in SST, were estimated using the formulation [9]

$$
\delta_{T I B L}=0.024 U \sqrt{\frac{T_{a} x}{\Delta T g}}
$$

with $U$ : wind speed, $T_{a}$ : air temperature, $x$ : downwind fetch, $\Delta T$ : the step decrease in sea surface temperature, and $g$ : gravitational acceleration. This formulation is only valid for the stable thermal layer, where $\Delta T>0$. In this work, this formulation is used at step changes from the warmer GS to cooler coastal water bodies. The fetch and step change in temperature are taken from SST imagery with direction from the SODAR based on the wind. We compare the height estimates to profiles of turbulence properties as validation.

In the analysis below, the section of the wind profile chosen for comparison with the MOST prediction is determined by the location of the buoy observations relative to the SST front. If buoy measured surface conditions indicate that the environment is unstable, the range of the wind profile selected for comparison is above the TIBL, within the unstable thermal layer, as pictured in Figure 4. Though the buoys are located on the shelf and therefore do not sample the Gulf Stream itself, instabilities on the shoreward side of the Gulf Stream produce warm filaments that often move warm water onto the shelf, especially in the study area of Raleigh Bay (e.g. Figure 1; [10]). If near surface buoy measurements indicate stable conditions are present, the range of the profile selected for analysis is within the stable thermal layer, but above the roughness layer - between the RIBL and TIBL heights. 


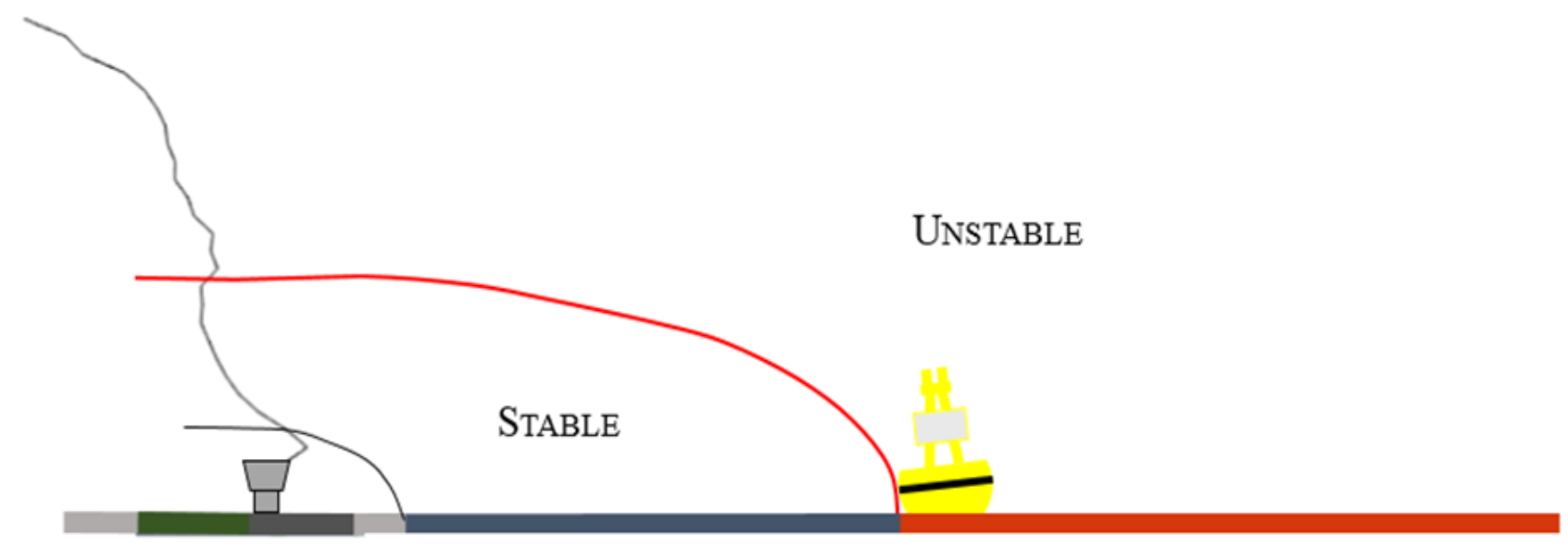

Figure 4 Visualization of IBL development over GS water (red), coastal water (blue) and land (gray). The height of the RIBL is depicted in black, beginning at Hatteras Island. The height of the TIBL is depicted in red, beginning at the horizontal SST step change at the cooler coastal water. These delineations show heights of the various IBL development in the wind profile over the SODAR. For this case buoy measurements are in warm water, where conditions are unstable.

\subsection{COARE 3.0}

To examine stability, an algorithm incorporating MOST, COARE 3.0, was used with environmental data from NDBC Stations \#41025 and \#41063. The buoys, as mapped in Figure 2, are located on the continental shelf near the Gulf Stream boundary and measure the necessary inputs to COARE, including air and water temperature, air pressure, humidity, and wind speed. COARE uses an iterative, flux-based method to estimate $L$, the buoyancy parameter known as the Obukhov length scale. $L$ can be taken as a quantification of the stability of the surface layer defined [11] as:

$$
L=-\frac{u_{*}{ }^{3}}{\kappa\left(\frac{g}{T_{0}}\right)\left(\frac{H_{0}}{\rho c_{p}}\right)}
$$

with the parameters $u_{*}$ : friction velocity, $k$ : von Karman constant, assumed 0.4 for this work, $g$ : gravitational acceleration, $T_{0}$ : surface air temperature, $H_{0}$ : surface kinematic heat flux, $\rho$ : density of the air, and $c_{p}$ : specific heat of air. The convention for a negative heat flux (heat flowing into the ocean) gives a positive value for $L$, indicating the atmosphere is stable with respect to the surface. COARE also estimates a value for friction velocity and aerodynamic roughness length, two parameters needed in conjunction with $L$ to model wind speed, $u$, as a function of height, $z$, in the logarithmic formulation

$$
u(z)=\frac{u_{*}}{\kappa}\left[\ln \left(\frac{Z}{z_{0}}\right)-\Psi_{m}\left(\frac{Z}{L}\right)\right]
$$

where $\Psi_{m}$ is the universal stability function. 


\subsection{Case Identification}

In order to best satisfy the steady-state, homogeneous flow assumptions within MOST, intervals of fairly consistent winds were identified, from 2013, the year when buoy observations upwind of the SODAR are available. Multiple time intervals of at least 15 hours in length were selected based on several criteria. The first entailed that the wind profile structure of each hourly measurement remained relatively similar throughout this time period. Values of $u_{*}$ obtained through logarithmic regression were scaled to mean speed and assessed for their magnitude and variation. Cases selected had scaled $u_{*}$ behavior that stayed between 0 and 0.5 for the majority of the time period. The second constraint for case selection was that the boundary layer growth rate as determined through the logarithmic fit was less than $40 \mathrm{~m} / \mathrm{hr}$. The final constraint, defining winds as coming from the ocean side of Hatteras, required that the wind direction stay within the range $110^{\circ} \mathrm{W}$ of $\mathrm{N}$ to $90^{\circ} \mathrm{E}$ of $\mathrm{N}$.

As the stability function is inversely related to $L$, a large value for this parameter yields little change to a wind profile as compared to the neutral case, where $\Psi_{m}=0$. The cases identified were further constrained to relatively steady environmental stability characteristics and classified based on the estimated mean value of $L$ : Strongly Unstable - SU, $-100<\bar{L}<0 \mathrm{~m}$; Weakly Unstable - WU, $1000<\bar{L}<-100 \mathrm{~m}$; Neutral $-\mathrm{N}, 1000<\bar{L}<-1000 \mathrm{~m}$; and Weakly Stable $-\mathrm{WS}, 0<\bar{L}<1000 \mathrm{~m}$. A total of ten cases were identified, spanning a range of stability characteristics, with widely varying profile characteristics (Figure 5). These ten cases are labelled and discussed in this paper according to these identifiers (e.g., strongly unstable case OSU1; weakly unstable cases OWU1, OWU2, OWU3, OWU4, etc.)

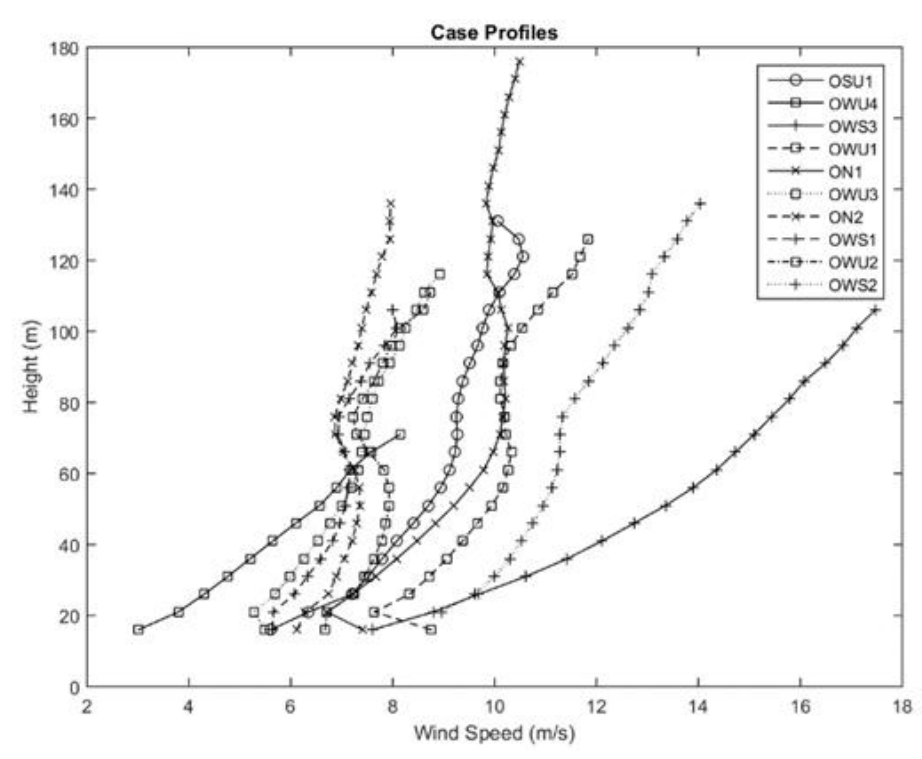

Figure 5 Time averaged wind speed profiles for each identified case, where $80 \%$ coverage is available.

A model utilizing the COARE output parameters was developed to assess the impact of the stability environment on a wind profile. The stability-based model is compared to a model assuming neutral conditions ( $L$ is infinite) and to the observations. The profile was constructed within a height range corresponding to the upwind environment where buoy measurements were taken. As noted above, when stable surface conditions were present, cases were modelled within 
the stable thermal layer, between the RIBL and TIBL heights. When unstable surface conditions were present, cases were modelled above the TIBL height.

These steps provide a method to use a stationary, land-based wind profile measurement to compare with the characteristics of the modelled wind profile at an upwind location if spatial conditions are well-defined. The results that follow will investigate the ability of the MOST-based model to accurately render the effect of stability on the profile as a whole and to provide an adequate estimate of the wind climate and wind resource in this coastal region.

\section{Results}

Three characteristics of the wind climate with relevance to wind energy engineering applications are mean wind speed, wind shear, and turbulence. The model developed through COARE was used to compare the ability of MOST to estimate wind speed and wind shear across the corresponding ranges of the wind profiles. The measured turbulence profiles from SODAR data (vertical velocity variance, $\sigma_{w}$, and backscatter intensity) were used to validate the IBL method through layer identification, and to comment on the general expectations of turbulence characteristics present within varying stability regimes.

The wind profiles identified within the ten steady-state case time periods exhibited widely varying levels of shear, independent of stability regime, with little similarity in structure on the whole (Figure 6). Wind shear can be large at low altitudes, or throughout the wind height range measured. One characteristic evident in several cases, and especially evident in the summer/fall profiles in Figure 6, is the low-level wind speed maxima, occurring around $50-70 \mathrm{~m}$ above the surface. These low-level maxima, or low-level jets (LLJs), are typically followed by lower wind speeds directly above, in addition to regions of high wind shear in the upper reaches of the measured profile. It can be seen through these mean wind profiles that wind shear is loosely tied to stability within a season, but the magnitude of wind shear can be high even in unstable cases.

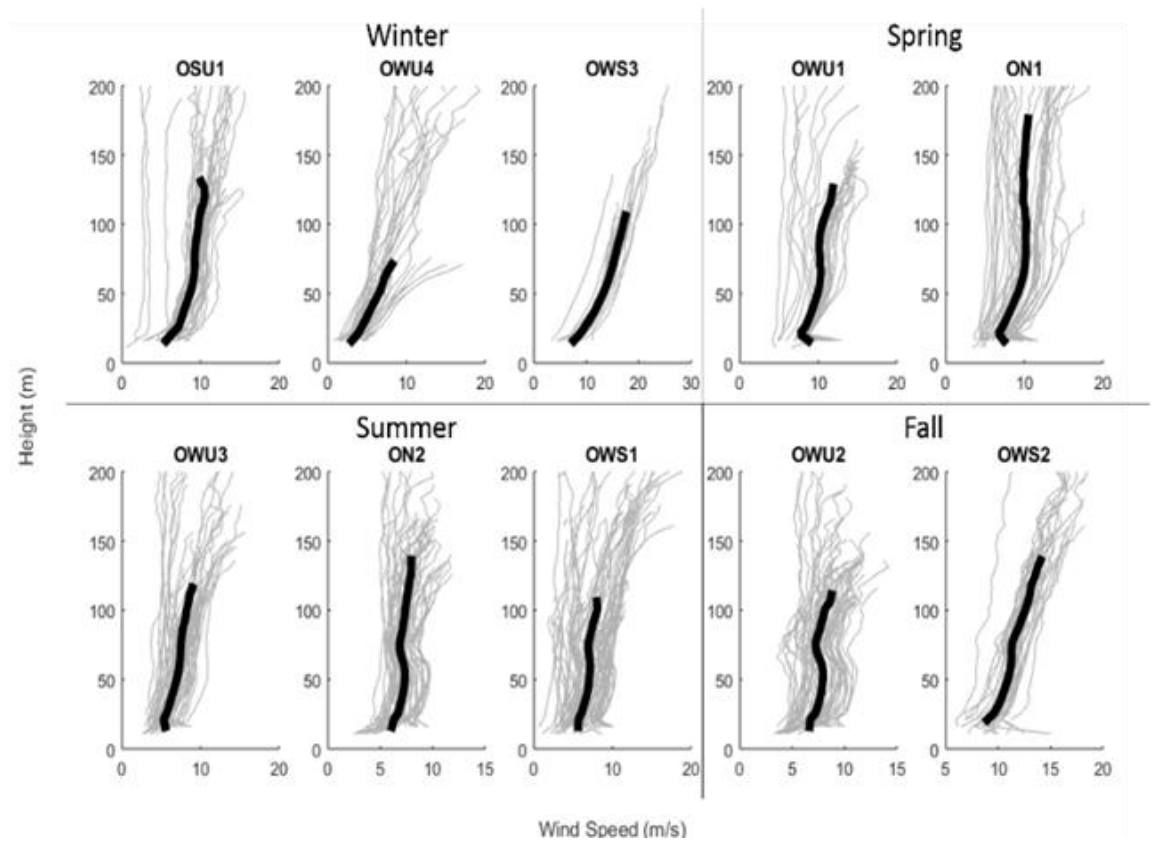

Figure 6 Mean wind profiles of each case (black), with hourly profiles shown in gray. Seasons are defined as: Winter (D, J, F); Spring (M,A,M); Summer (J,J,A); Fall $(S, O, N)$. 


\subsection{Stable Cases}

COARE-based hourly wind profiles are compared with observations for the weakly stable case, OWS2 (Figure 7, upper panel). The TIBL is a characterizing influence here: shear remains consistent and large throughout the comparison range. In the majority of the hourly profiles, the shear is well resolved; however, the shear is underestimated during a considerable time interval at the beginning of the case. The wind speed, however, is overestimated by 1-3 m/s for all but one hour. As can be seen in Figure 8, this overestimation in mean wind speed is a characteristic of the model for every stable case. This "up-shift" in estimated wind speeds, especially obvious where the wind shear is well resolved, but wind speed is overestimated, will be addressed in the discussion section. The wind shear was underestimated when modelled assuming neutral stability but the mean speed more accurately represented the observations (Figure 8). Addition of the stability parameter within the model generally improved the wind shear estimation though sometimes the estimate was still relatively low (OWS3).
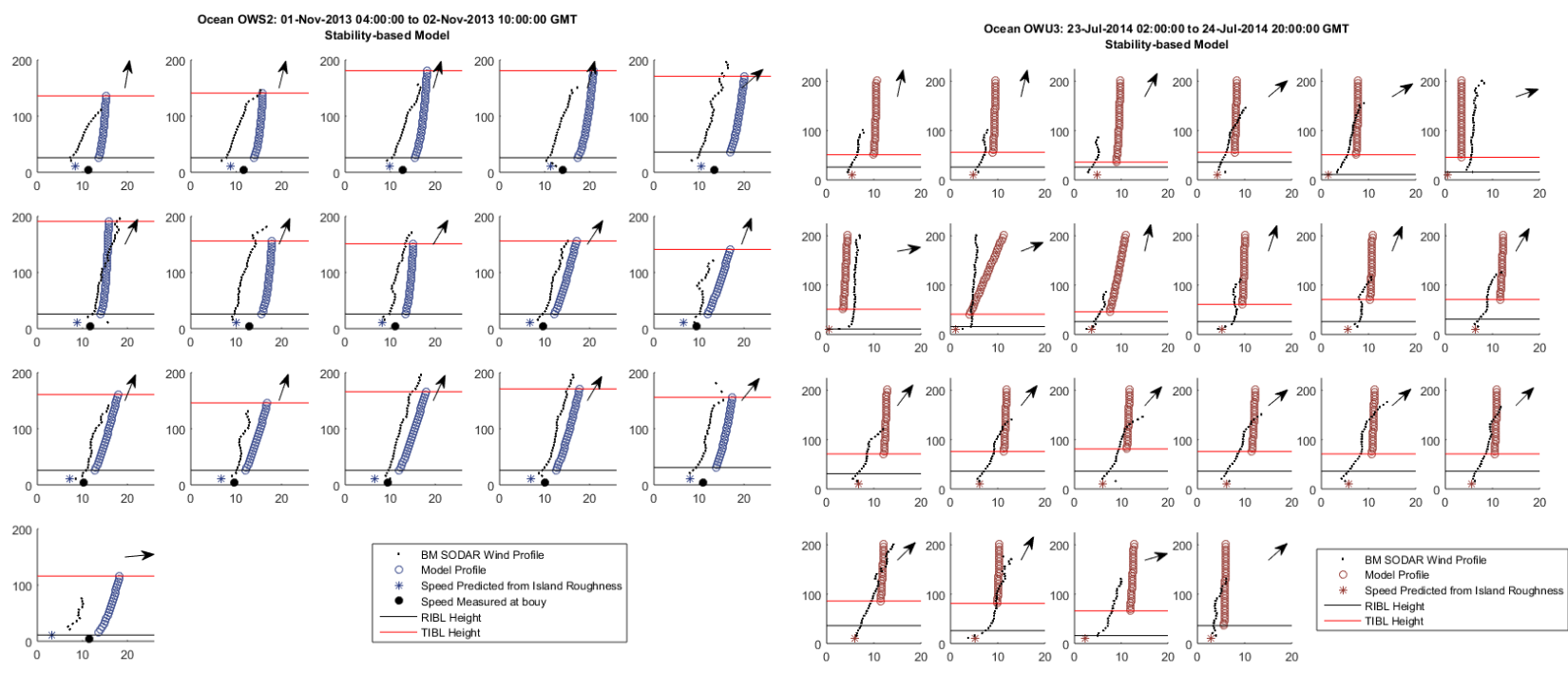

Figure 7 Stability-based model results, shown in hourly increments for OWS2 (left panel) and OWU1 (right panel). The model profile is formed in the relevant range for each case - between the RIBL and TIBL for the stable case, and above the TIBL for the unstable case. The measured wind profile for comparison, with the wind direction indicated by the arrow in the upper right of each figure. 

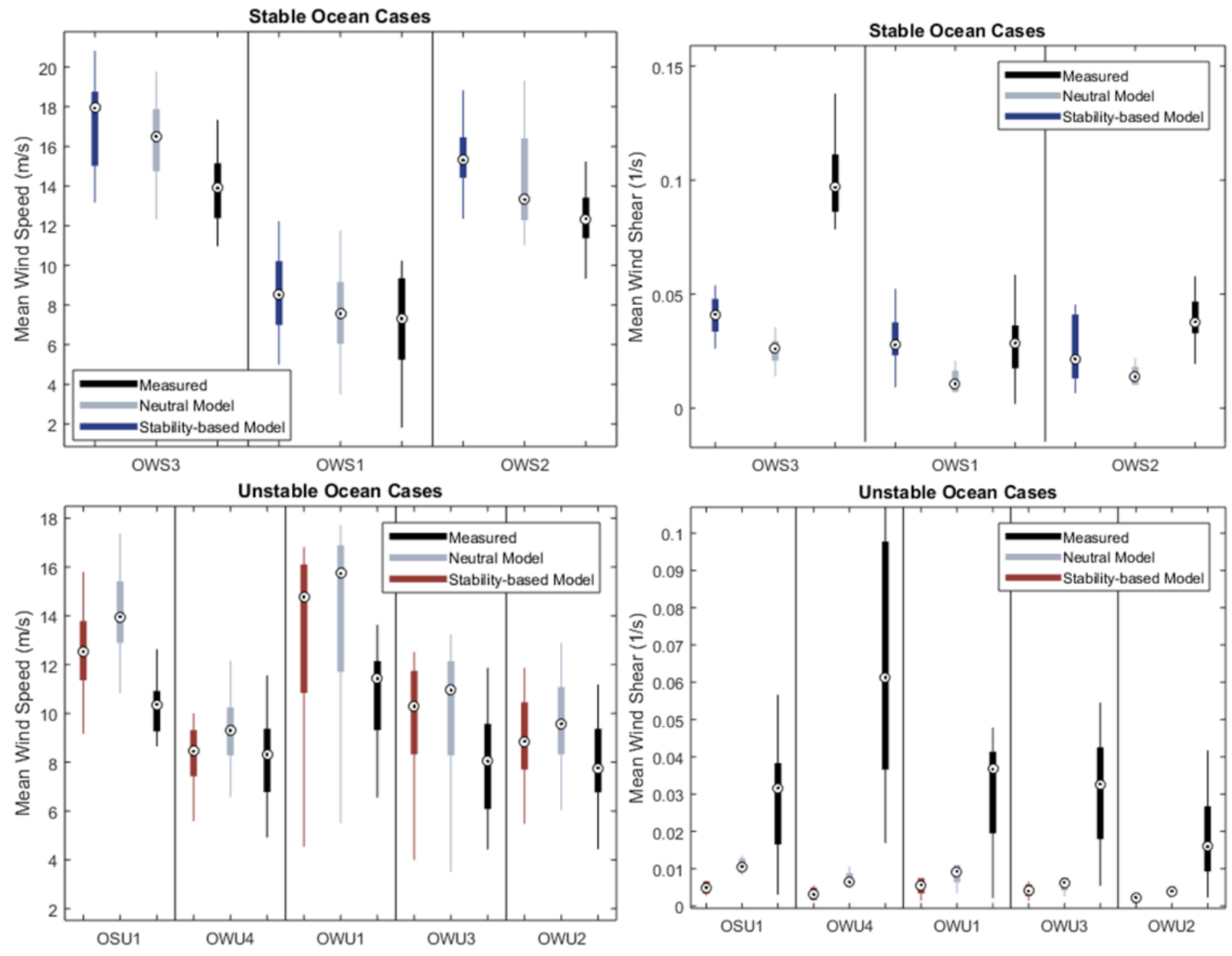

Figure 8 Values of height-averaged wind speed (left panels) and wind shear (right panels) in stable cases (top row) and unstable cases (bottom row). The variation within each case is shown through the boxplots of the modelled and measured values.

\subsection{Unstable Cases}

Cases that were classified as unstable were generally poorly modelled using a stability-based model (one case shown in full in Figure 7, lower panel). Modelled mean wind speed was again too large, though to a lesser degree than for the stable cases. The stability correction reduced the misfit relative to the neutral model. Observed wind shear was greatly underestimated by COARE: observed wind shear above the TIBL can be high, especially in cases where the wind profile exhibits the LLJ shape, with greater shear above the jet. As can be seen in Figure 8, the wind shear was badly underestimated assuming neutral conditions (in gray). A stability-based model in unstable conditions, where $L<0$, decreased shear and only worsened the comparison to the observations. The unstable formulation used in the ocean cases exacerbated the already underestimated wind shear from the neutral profile.

A root mean square error was formed of deviations from the mean for both the neutral model and stability-based model $M$ and the measured wind profile $S$ as

$$
\begin{aligned}
M_{j k}^{\prime} & =\overline{M_{k}}-M_{j k} \\
S_{j k}^{\prime} & =\overline{S_{k}}-S_{j k}
\end{aligned}
$$




$$
R M S_{i}=\sqrt{\frac{\sum_{j=1}^{t_{i}} \sum_{k=1}^{z_{j}}\left(M_{j k}^{\prime}-S_{j k}^{\prime}\right)^{2}}{t_{i} \sum_{j=1}^{t_{i}} z_{j}}}
$$

where $t_{i}$ represents the number of hours in the case, $z_{j}$ represents the number of relevant measurements in the vertical, $k$ is each wind profile height, and $j$ is each profile in time. RMS values are shown in Figure 9. The stable cases were either unchanged or slightly improved by including the stability parameter, but unstable cases were usually worsened. The RMS values provide a measure of the success the model has in predicting the shape of the wind profile, and in adequately estimating wind shear across a range of heights, and demonstrates that uncertainties are $0.5-2 \mathrm{~m} / \mathrm{s}$ for hourly values.

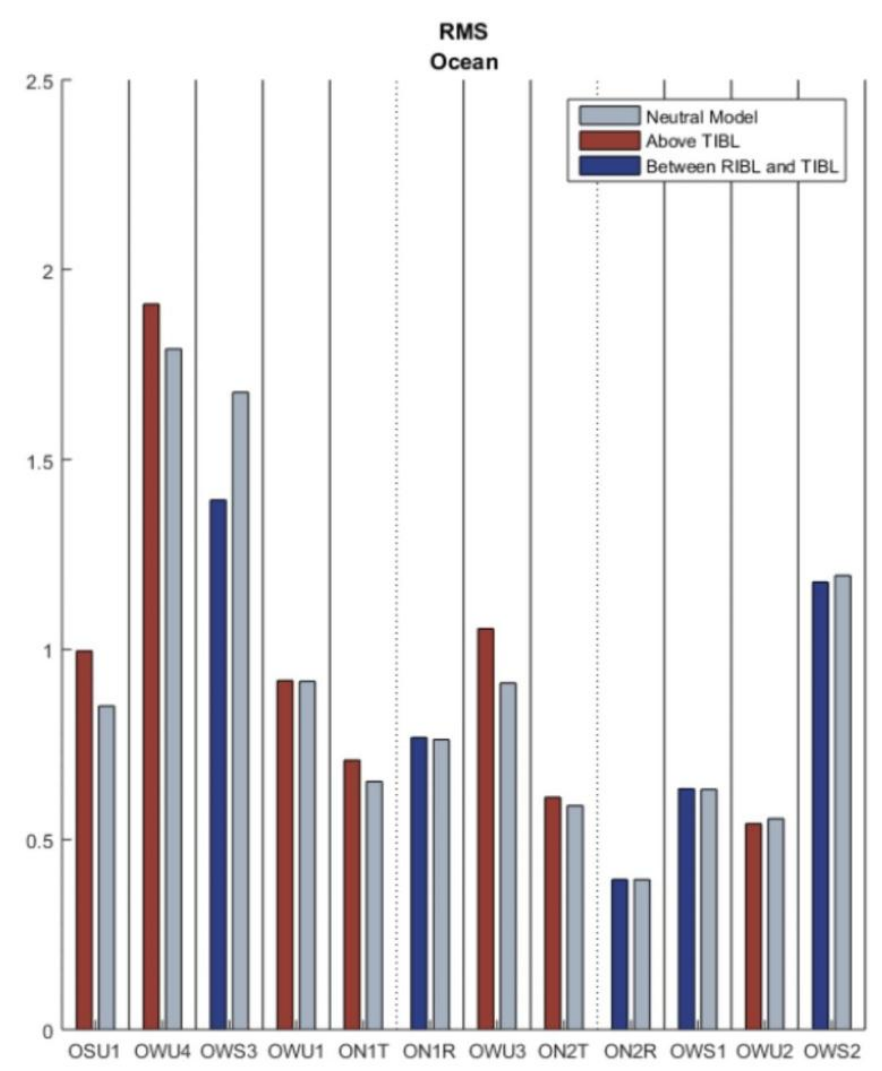

Figure 9 RMS values (in $\mathrm{m} / \mathrm{s}$ ) for each case. Neutral cases were formed against each layer of the wind profile (R: RIBL to TIBL, T: above TIBL) for comparison purposes.

\subsection{Turbulence Validation of IBL Method}

The IBL height approximations were conducted using rough estimations of horizontal heterogeneity at ground level. The validity of these heights can be investigated by comparing them to the turbulence profiles provided by the SODAR [12]. Knowledge of turbulence behaviour is also necessary for wind energy engineering especially in areas of high variability - for both power curve estimations and evaluations of the material stresses on turbine blades. High levels of turbulence can cause unexpected effects [13]. Especially in an unstable layer, turbulence measurements remain of interest despite the characteristically low levels of wind shear. The unstable regime is a convective region of the atmospheric boundary layer. While wind shear may 
remain low, turbulence can be unexpectedly high, especially if neutral stratification is assumed based solely on wind profile measurements. It has been shown that the vertical distribution of turbulence can be characterized as a "traditional" boundary layer with upward transport of turbulent kinetic energy (TKE), or as an "upside-down" boundary layer, with downward transport of TKE [14]. The velocity variance profiles either decrease or increase with height, respectively, in these systems.

In the weakly stable case (OWS3) presented in Figure 10, the vertical velocity variance indicates that an "upside down" boundary layer begins at around the same height as the top of the TIBL. Assuming the vertical velocity variance is representative of turbulent mixing intensity, this finding corroborates the TIBL height estimate by suggesting a downward turbulent transport above the stable layer associated with warmer, Gulf Stream waters. It is worth noting that the "traditional" boundary layer form is rarely observed through these vertical velocity variance measurements. This may indicate that in this location during onshore winds with a region of stable stratification above the sea surface downward turbulence flux is a more characterizing influence on the layer from sources aloft in the ABL than is the upward transport of surface heat sources.

The top of the RIBL appears to correspond to a maximum in backscatter intensity. The top of the RIBL could be a region of the wind profile with more energetic small scale turbulence as a result of mixing between the two distinct layers. These turbulence measurements provide some confirmation that the IBL algorithms developed do provide reasonable estimates of the heights of significant change in the wind profile. The stable layer in particular is well reasoned through these turbulence measurements - regions of low vertical velocity variance within correspond well with TIBL height estimations.

OWS3
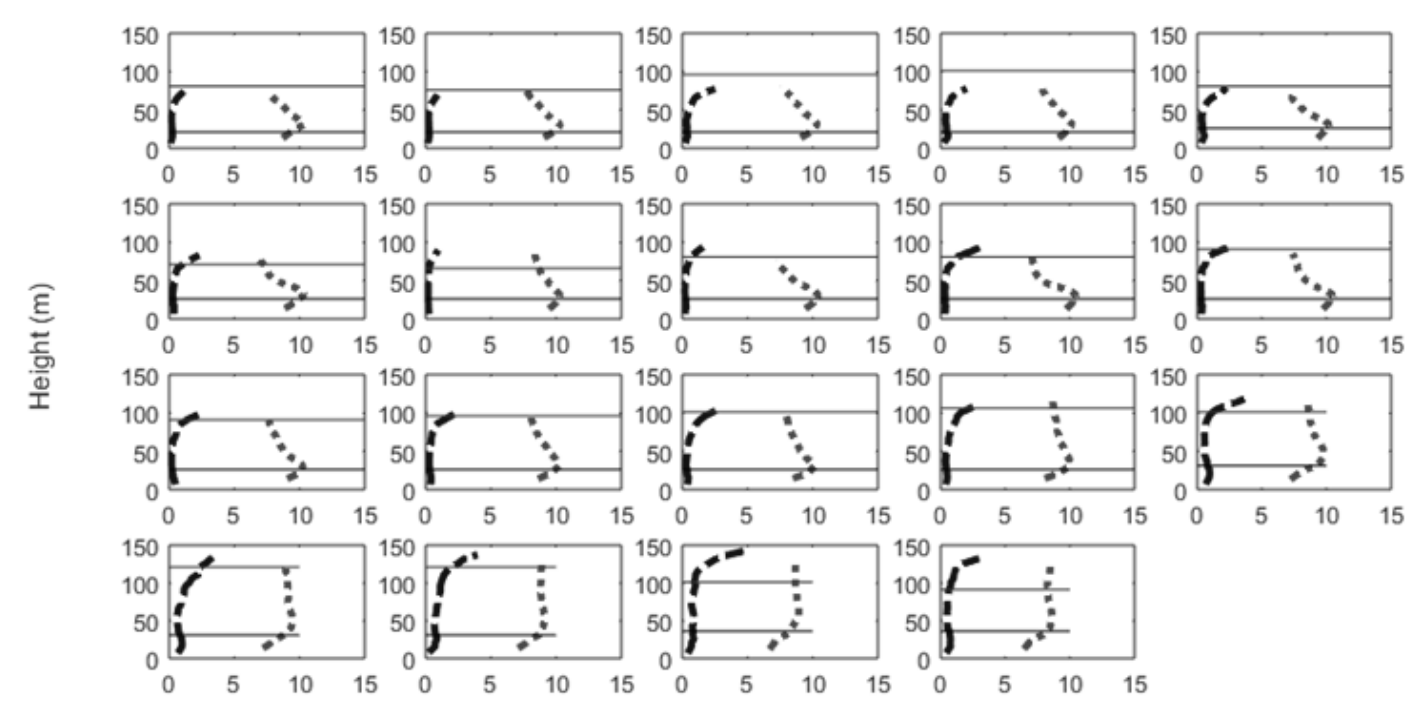

$-\mathbf{- n}-10^{*} \sigma_{\mathrm{w}}^{2}$

-......Backscatter Intensity $(\log )$

Figure 10 Turbulence measurements (vertical velocity variance and backscatter intensity) shown as height profiles in OWS3 case. Horizontal lines mark the height of the RIBL and TIBL. 


\section{Discussion}

\subsection{Model Limitations in This Area}

Heterogeneities present in the area offshore of North Carolina present several difficulties in applying MOST to the available datasets. The environmental measurements used to calculate $L$, and classify the cases in terms of stability, were obtained from buoys offshore of North Carolina on the continental shelf. The dynamic nature of the thermal intrusions from the Gulf Stream means that the buoys may be situated in a region of the ocean that is changing rapidly with respect to SST, and consequentially rapidly changing lower atmosphere surface layer stability conditions, even within the time period of an identified case. The spatial heterogeneities inherent to this location make the stability of the lower atmosphere transient temporally and geographically. The $L$ value estimated through COARE could (1) be poorly representative of the conditions encountered by the wind measured at the SODAR, or (2) could accurately represent the conditions, but at a time significantly different from when the profile measurement was taken. A simple estimate of time-lag between the locations (around 0-2 hours depending on wind speed and buoy used), suggests the lag should not greatly impact the hourly resolved modelled wind profiles. That such a lag is not visually apparent in the hourly resolved models (e.g. Figure 7) suggests that while spatial separation may be an influencing factor, alone it is not sufficient to explain the failures of the MOST method in general. These possibilities are indicative of a larger problem - the assumptions within MOST include a flow being both stationary and homogenous, two characteristics that are rarely present in a geophysical environment, especially one as dynamic as the region near Cape Hatteras and the GS. The measured profiles, while under relatively steady conditions, may contain remnants of in-homogenous flow, violating MOST assumptions especially as the case initializes. This trait could be evidenced in the accuracy of the model improving throughout the case. As seen in the stable case in Figure 7, the model more accurately renders wind shear in the latter half of the case. That is to say, as the directionality and stability conditions have the chance to affect the wind profile steadily, the MOST expectations can be well met.

Yet another surprising finding is the high baseline shear found across all seasons and all stability conditions. Ranges exhibiting high shear are consistently found high in the wind profile, in what should be an unstable thermal layer, as seen in a typical wind profile shown in Figure 11. Within the stable thermal layer, a low-level maxima is present - a characteristic typical of stable surface layers. Above the TIBL height, however, in a region of the ABL assumed to be unstable and associated with upwind conditions over the Gulf Stream, the wind profile also exhibits high shear. The model does not correctly represent this shear, as environmental conditions imply an unstable regime, where low shear and high turbulence is expected. This geographical region may be influenced by environmental factors unincorporated in COARE or MOST empiricisms that result in high shear even within unstable conditions. These results are especially interesting when coupled with the turbulence data - there is typically a steep increase in vertical velocity variance above the estimated TIBL height. The downward turbulence transport implied by this profile shape suggests that the unstable layer may be downwardly convective, and more strongly influenced by sources aloft in the $A B L$ [15]. In the interstitial region between the stable and unstable layers, the flux divergence accompanying changes in stratification characteristics may explain the unexpectedly 
high levels of shear within the unstable layer. The vertical coverage of SODAR measurements may not extend into the true "free convective" layer where wind speeds are more consistent with height. This interstitial region of significant momentum flux, may be a substantial vertical range up to $100 \mathrm{~m}$ in scale. The unstable conditions observed over the Gulf Stream may initiate a convective, low wind shear region of the surface layer, but above the stable and interstitial regions beyond the range of the SODAR as used in this study.

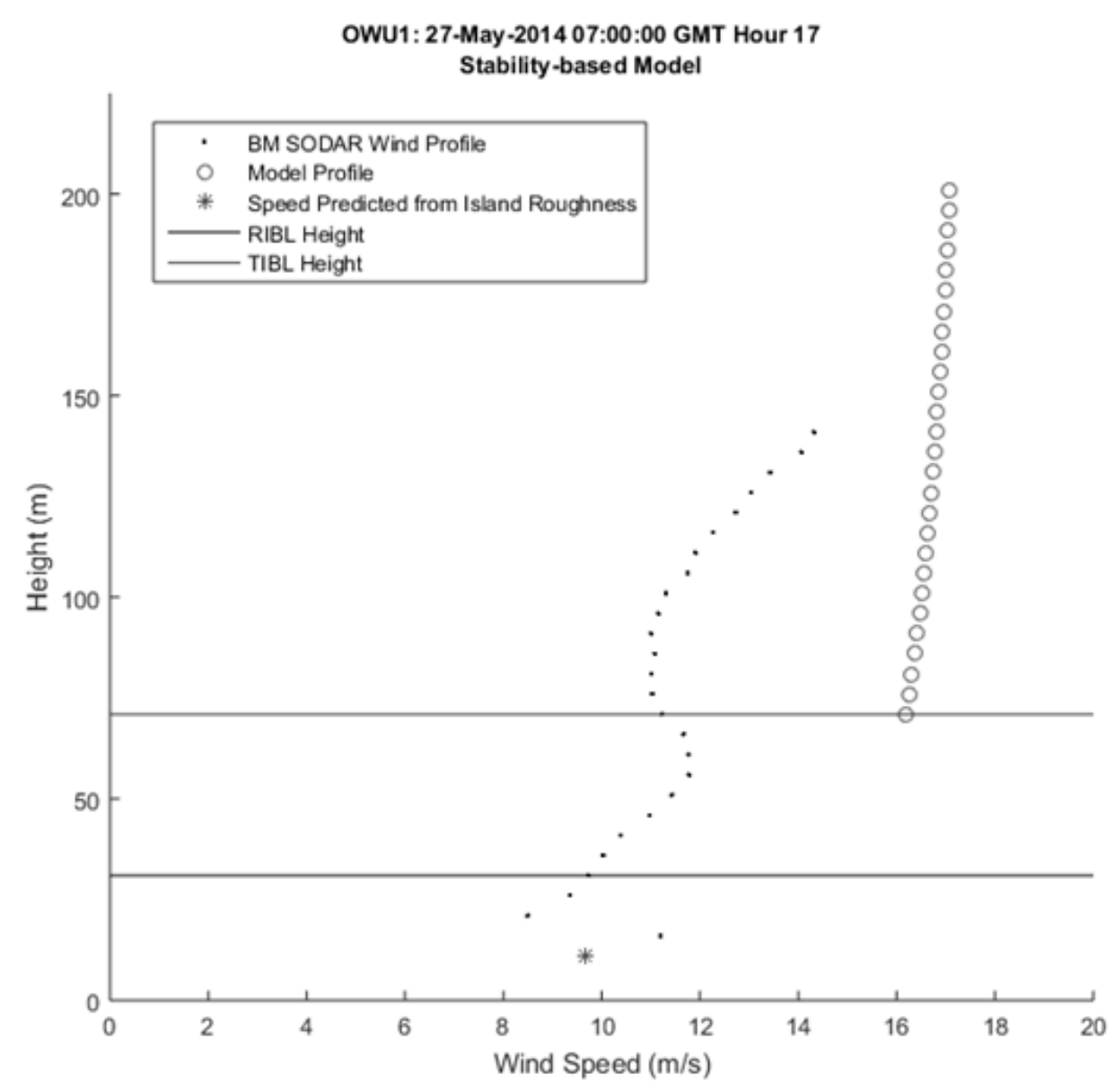

Figure 11 OWU1 hourly wind profile and model result.

\subsection{Over-Prediction of Wind Speed}

Wind speed estimation is a factor of interest both in understanding environmental effects on wind profiles, as well as for broader implications of wind resource estimation. The stability-based model consistently under-estimated wind shear, especially for unstable cases, but tends to be more accurate in stable cases, evidenced in Table 1. Wind speed, however, was generally overestimated by roughly $20 \%$ (by $1.91 \mathrm{~m} / \mathrm{s}$ in the mean), even in hours where wind shear was well resolved. This "up-shift" in the wind profile could be an effect of surface roughness and form drag being inadequately incorporated into the model. When wave heights, wave periods, and current speeds were included in COARE in those cases where data was available, these characteristics did not account for the overestimation in wind speeds. Additionally, the sea state at the buoy, while possibly not in equilibrium with the wind because of the continental shelf depths $(30 \mathrm{~m})$, is at greater surface equilibrium with the wind climate than the near-shore 
environment. Wave action and shoaling downwind of the buoy can be expected to have effects on near surface winds within the TIBL. While the aerodynamic roughness is explicitly estimated through COARE, geometric surface roughness has also been shown to increase with wind speed at these scales [16]. Form drag of the lower $A B L$ could be influencing the wind profile over these fetches, decreasing wind speed as it approaches and crosses the coastline.

Table 1 Comparison of measured and MOST modeled mean wind. Difference is reported as measured-predicted, with a positive convention indicating an underestimation.

\begin{tabular}{lllll}
\hline & \multicolumn{2}{l}{ WIND SPEED $(\mathrm{m} / \mathrm{s})$} & \multicolumn{2}{l}{ WIND SHEAR $(\mathbf{1} / \mathrm{s}) * 1 \mathbf{0}^{\mathbf{2}}$} \\
\hline Cases & Mean & Mean & Mean & Mean \\
& Measured & $\begin{array}{l}\text { Modeled } \\
\text { Difference }\end{array}$ & Measured & $\begin{array}{l}\text { Modeled } \\
\text { Difference }\end{array}$ \\
All & 9.52 & -1.91 & 4.02 & +2.76 \\
Stable & 11.09 & -2.59 & 5.74 & +2.54 \\
Unstable & 8.88 & -1.58 & 3.80 & +3.44 \\
\hline
\end{tabular}

\subsection{Wind Resource Implications}

Thomas et al. [17] used measurements of the difference between SST and surface air temperature (SAT) to incorporate stability information into wind energy area resource estimation in coastal North Carolina. The findings from the present work, including slower than predicted wind speeds, high baseline shear measured by the SODAR, and slow stable thermal layer growth rates, suggest that applying MOST is questionable in some settings, especially where large SSTs changes occur. It appears that the mean speed in the IBLS is less than expected, at least by the time it was measured over land, which is inconsistent with simple momentum exchange between layers. This implies an additional source of drag that cannot be accounted for in a simple 1D formulation such as MOST. A likely candidate is form drag, which can only be represented in a spatially-explicit model.

High wind shear is present throughout the range of measured wind profiles, regardless of season, or stability regime. The high shear above the stable thermal layer suggests that high shear is present even in the lower portion of unstable layers originating above GS waters once they are detached. While SST-SAT information indicates that this region is generally unstable in the surface layer, the high wind shear findings indicate that the environment is more complicated, and a high baseline shear exists in the region, even in environments where convective regimes are expected. This finding, supported by the combination of wind speed profile measurements, and the turbulence profiles of the SODAR, indicate that high turbulence and high shear may coexist in regions as thermally dynamic as this one. In contradiction to recent findings in the North Sea, where it has been shown that turbulence and high wind shear are characteristic of unstable and stable conditions, respectively [18], our measurements indicate that wind energy developers in this area may need to account for both high shear and high turbulence in power, load, and fatigue estimates. While a distinct TIBL marks regions of higher or lower turbulence, it is clear that these 
characteristics are not always easily predicted given previous knowledge of atmospheric stability influences alone.

Further, this result may suggest that a TIBL algorithm could be useful in wind energy development, in searching for regions far enough away from horizontal thermal gradients that high turbulence may be avoided. It is worth noting, however, that the "upside-down" boundary layer identified by [13] is also characterized by a low-level wind maximum above the turbulence region. If wind turbine structures are able to withstand higher levels of turbulence structurally and maintain generation capabilities, the capacity payoff may be worthwhile. These stable thermal layers grow slowly when compared to unstable thermal layers [19]. The cooler coastal waters are typically classified as stable through SST-SAT differences, and the slow growth of the stable layer shows that the effects on the wind profile from this stratification might not reach wind turbine hub height in short time periods, or short fetches. It is not obvious how these effects impact the wind resource but do suggest a number of uncertainties that need to be addressed.

\subsection{Suggested Next Steps}

Based on the results of this study we suggest possible next steps to improve upon our understanding of the wind resource offshore of North Carolina. These results highlight the need for robust data collected within the marine environment. One obvious need is wind profile observations over the coastal ocean. A higher range of coverage would also be valuable - to identify the free convective layer, if it exists, and verify where the high shear region ends. An ability to collect information about atmospheric stability at these marine sites is also critical. Were these to be made simultaneously with wind profile observations at Billy Mitchell airport, with appropriate ancillary observations of the stability conditions, it should be possible to quantify any change in wind speed within IBLs. Should offshore wind be more consistent with MOST predictions, the paired data would permit an estimate of the form drag. A more spatially explicit estimation of TIBL growth rates should be included in future analyses to determine variations in the height to which atmospheric stability will influence the $A B L$, and therefore the wind characteristics at hub height. This would be most easily accomplished with a primitive equation model, such as WRF, though it would likely require coupling to a regional ocean model to provide appropriate lower boundary conditions.

\section{Conclusions}

The results discussed above reveal that the MOST based COARE algorithm is useful in determining the wind profile structure in coastal environments, despite being developed for the open ocean. The shortcomings in estimating wind speed, wind shear, and in modelling the wind profile could be as much an effect of insufficient or unsuitable datasets as of inadequacies in the theoretical application. Mean wind speed estimations were on the order of measured values, but were consistently overestimated in stable and unstable ocean cases. This finding is interesting when coupled with the RMS error results, where it was indicated that the profile shape of stable ocean cases is better resolved. In all cases, wind shear was underestimated assuming neutral stability, and only estimations within stable environments improved when incorporating a stability parameter into the model. Wind shear is consistently higher than expected, especially in unstable conditions. A more accurate understanding of the mechanisms driving wind shear in the region 
should be addressed, as these greater values of shear could be a particular concern to offshore wind development. Finally, analysis of velocity variance and backscatter measurements throughout the wind profile were useful in validating simple formulations of IBLs and their relationship to turbulence that could be of use to wind farm development and operations.

Overall, results from this study suggest that knowledge of stability can improve wind-at-height estimations when environmental conditions like air-sea heat exchange processes, thermal structure, and surface wind characteristics, are well known, but wind shear in this region is typically underestimated with MOST. A better understanding of the conditions that cause this high baseline shear (present in unstable, neutral, and stable conditions) is essential for anticipating changes in shear with stability, as well as the associated impact on wind-at-height estimations. The wind resource could be better characterized in coastal North Carolina, and potential lease blocks could be better identified, by using information about the spatial structure of thermal influences like the Gulf Stream, and the cooler coastal waters of the Mid- and South Atlantic Bights. However, a spatial characterization of this influence should account for the slowly-growing stable layer there may be regions with stable atmospheric conditions where the surface layer has not grown to turbine hub-height, and the expected change in wind speed and shear would not yet be present. Future analysis of the available wind resource should include offshore wind profile observations, and directionally explicit estimation of thermal layer growth rates, to determine the height to which influences of atmospheric stability will influence the ABL. It is clear that measured wind profiles over the ocean are necessary to more confidently assess wind speeds and the levels of shear in this region.

\section{Acknowledgments}

We acknowledge Sara Haines from UNC Chapel Hill for leading the buoy development effort and leading the data management from all instrumentation, and Mike Muglia with the Coastal Studies Institute for supporting and maintaining the SODAR and buoy installations, and Dr. John Bane for insightful discussions about internal boundary layers.

\section{Author Contributions}

Schutt conducted the majority of the analysis and the writing of the manuscript. Seim aided in interpretation of the results and in the writing of the manuscript.

\section{Funding}

We gratefully acknowledge the North Carolina Department of Commerce State Energy Office which provided initial support for the SODAR and its deployment, Duke Energy for support for the extended operation of the SODAR, one of the offshore buoys, and the authors, and to Duke Energy Fellowship Program of the University of North Carolina Institute for the Environment which provided support for Schutt.

\section{Competing Interests}

The authors have declared that no competing interests exist. 


\section{References}

1. Lantz E, Wiser R, Hand M. IEA Wind Task 26: The Past and Future Cost of Wind Energy. Golden, CO: National Renewable Energy Laboratory; 2012.

2. Holtslag MC, Bierbooms WAAM, van Bussel GJW. Validation of surface layer similarity theory to describe far offshore marine conditions in the dutch North Sea in scope of wind energy research. J Wind Eng Ind Aerod. 2015; 136: 180-191.

3. UNC. Coastal wind: Energy for North Carolina's future. Rep. n.p.: UNC; 2009.

4. Bureau of Ocean Energy Management. Announcement of area identification: Commercial wind energy leasing on the outer continental shelf offshore North Carolina. BOEM. n.p.; 2014.

5. Mahrt L, Vickers D, Moore E. Flow adjustments across sea-surface temperature changes. Bound Lay Meteorol. 2004; 111: 553-564.

6. Fairall CW, Bradley EF, Hare JE, Grachev AA, Edson JB. Bulk parameterization of air-sea fluxes: Updates and verification for the COARE algorithm. J Climate. 2003; 16: 571-591.

7. Rutgers University Sea Surface Temperature Satellite Imagery main web page: https://marine.rutgers.edu/cool/sat data/?product=sst\&nothumbs=0.

8. Stull RB. An introduction to boundary layer meteorology. Dordrecht: Kluwer Academic; 1988.

9. Garratt JR. The atmospheric boundary layer. Cambridge: Cambridge University Press; 1992. pp. 186-191.

10. Glenn S, Ebbesmeyer K. Observations of Gulf Stream frontal eddies in the vicinity of cape hatteras. J Geophys Res. 1994; 99: 5047-5055.

11. Foken T, Nappo CJ. Micrometeorology. Berlin: Springer; 2008. pp. 55.

12. Emeis S. Wind energy meteorology. Berlin: Springer; 2013. pp. 179-182.

13. Honrubia A, Vigueras-Rodrigues A, Gomez-Lazaro E. The influence of turbulence and vertical wind profile in wind turbine power curve. Prog Turbul Wind Energ. 2012; 141: 251-254.

14. Mahrt L, Vickers D. Contrasting vertical structures of nocturnal boundary layers. Bound Lay Meteorol. 2002; 105: 351-363.

15. Banta RM, Pichugina YL, Brewer WA. Turbulent velocity-variance profiles in the stable boundary layer generated by a nocturnal low-level jet. J Atmos Sci. 2006; 63: 2700-2719.

16. Kudryavtsev VN, Makin VK. Aerodynamic roughness of the sea surface at high winds. Bound Lay Meteorol. 2007; 125: 289-303.

17. Thomas N, Seim H, Haines S. An observational, spatially explicit, stability-based estimate of the wind resource off the shore of North Carolina. J Appl Meteorol Clim. 2015; 54: 407-425 (150828110930006).

18. Holtslag MC, Bierbooms WAAM, van Bussel GJW. Far offshore wind conditions in scope of wind energy. TU Delft. 2016. Available from: https://repository.tudelft.nl/islandora/object/uui d:3c66f401-6cff-4273-aa49-df4274ba767f?collection=research.

19. Garratt JR. The internal boundary layer - A review. Bound Lay Meteorol. 1990; 50: 171-203. 
JEPT 2020; 2(2), doi:10.21926/jept.2002006

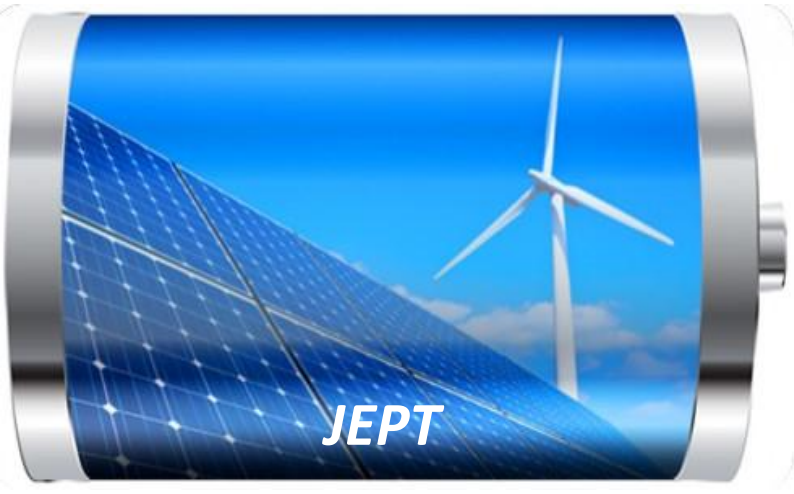

Enjoy JEPT by:

1. Submitting a manuscript

2. Joining in volunteer reviewer bank

3. Joining Editorial Board

4. Guest editing a special issue

For more details, please visit:

http://www.lidsen.com/journal/jept 